\title{
An Unexpected Case of Cannabis-Induced Pancreatitis
}

\author{
Fiona Lubega ${ }^{1}$, Anita Lwanga ${ }^{2}$ \\ 1. Internal Medicine, Saint James School of Medicine, The Quarter, AIA 2. Internal Medicine, Samaritan Medical Center, \\ Watertown, USA
}

Corresponding author: Fiona Lubega, filubega@gmail.com

\begin{abstract}
As cannabis becomes legalized in many jurisdictions around the world, its popularity is increasing. Despite the presence of several publications reporting cannabis-induced acute pancreatitis, the mechanism and pathophysiology of this entity are poorly understood. This case report highlights the course of a 28 -year-old male who presented to the hospital with complaints of abdominal and back pain. Because his lipase was elevated, he met the revised Atlanta criteria for a diagnosis of mild acute pancreatitis. After excluding more common causes, it was determined that his pancreatitis was likely due to cannabis use. This case adds to the body of research reporting on cannabis-induced acute pancreatitis. In the future, we hope to see strict diagnostic criteria and further research to elucidate the mechanism and pathophysiology of this entity.
\end{abstract}

Categories: Internal Medicine, Medical Education, Gastroenterology

Keywords: acute pancreatitis, cannabis, cannabinoids, tetrahydrocannabinol, the

\section{Introduction}

Cannabis is the most widely used recreational drug worldwide, with over $4 \%$ of the world's population using it annually [1-2]. It has been used to treat nausea and vomiting secondary to chemotherapy, abdominal pain, cancer-related pain, irritable bowel syndrome, and to reduce the symptoms of acute inflammatory bowel disease [3]. Paranoia, psychosis, infertility, and visual changes are the documented side effects of cannabis use $[1-2,4]$.

Common causes of acute pancreatitis include gallstones, alcohol abuse, smoking, hypertriglyceridemia, infections, trauma, drugs, malignancy, scorpion stings, hypercalcemia, and endoscopic retrograde cholangiopancreatography (ERCP) [1-2,5-6]. Opioids, angiotensin-converting enzyme (ACE) inhibitors, macrolides, nonsteroidal anti-inflammatory drugs (NSAIDs), diuretics, statins, and cannabis have been associated with acute pancreatitis $[2,5]$. While prescription medications account for $2 \%$ of cases of acute pancreatitis, cannabis use accounts for even fewer cases [2].

Review began 01/13/2021 Review ended 02/05/2021 Published 02/10/2021

๑) Copyright 2021

Lubega et al. This is an open access article distributed under the terms of the Creative Commons Attribution License CC-BY 4.0., which permits unrestricted use, distribution, and reproduction in any medium, provided the original author and source are credited.
In the first reported case of cannabis-induced pancreatitis, which was published by Grant and Gandhi, a challenge posed to clinicians was the patient's hesitation to disclose his cannabis use because of issues surrounding the legality of the substance [1]. Restrictions on cannabis use have since been lifted; this raises the possibility for further exploration of this topic [1]. We report a rare case of cannabis-induced pancreatitis, which we hope will add to the body of research supporting the presence of this entity.

\section{Case Presentation}

A 28-year-old male presented to the hospital with complaints of nausea, vomiting, and severe lower abdominal pain that radiated to his back. He denied having fevers, chills, constipation, or diarrhea. The patient had a medical history of diverticulitis and migraines. He did not have a family history of medical problems, did not have allergies, and did not take any medications. He did not use alcohol or tobacco products but admitted to using cannabis.

The patient's temperature was $99.2^{\circ} \mathrm{F}$, heart rate was 77 beats per minute, respiratory rate was 20 respirations per minute, and his blood pressure was $142 / 83 \mathrm{mmHg}$. His abdomen was flat, he did not have Cullen's sign or Grey Turner's sign, bowel sounds were hypoactive, and he complained of tenderness with palpation of the lower abdomen. The rest of his physical exam was unremarkable. His white cell count was $14,700 / \mu \mathrm{L}$ and a serum lipase was 1,022 U/L. The rest of his complete blood count, serum electrolytes, serum calcium, serum creatinine, serum urea, aspartate aminotransferase, alanine aminotransferase, alkaline phosphatase, lactate dehydrogenase, serum bilirubin, cholesterol, and triglycerides were within their reference range. His serum ethanol was negative, but his urine drug screen was positive for delta-9tetrahydrocannabinol (THC) and opiates. Both the computed tomography (CT) scan of the abdomen and pelvis and ultrasound of the liver and gallbladder were unremarkable.

The patient's complaints of abdominal pain and presence of lipase that was elevated more than three times 
the upper limit of normal, met the revised Atlanta Criteria to diagnose mild acute pancreatitis [7]. The patient's medical and social history ruled out tobacco abuse and prescription medications as possible causes but raised the possibility of cannabis-induced pancreatitis. The blood tests and imaging studies ruled out gallstones, alcohol, IgG4-related disease, or hyperlipidemia as possible causes of acute pancreatitis. His urine drug screen was positive for opiates because he received morphine in the emergency room to manage his pain prior to collecting a urine sample. The presence of THC in the urine drug screen further affirmed the possibility that the pancreatitis was due to cannabis use.

To help confirm the possibility of cannabis-induced pancreatitis we used the Naranjo Nomogram for Adverse Drug Reaction Assessment. The patient's Naranjo score was 6, which corresponds with a rating of probable adverse drug reaction. This confirmed cannabis as the probable cause of his acute pancreatitis. Initially, the patient was instructed not to eat and received intravenous fluids along with intravenous Toradol and morphine to manage his abdominal pain. His abdominal pain improved prior to discharge from the hospital and he was instructed to follow up with his primary care provider. Fortunately, he has not been readmitted to the hospital.

\section{Discussion}

Cannabinoid receptor 1 (CB1) and cannabinoid receptor 2 (CB2) are the two main types of cannabinoid receptors in the human body [3,6,8-9]. While CB1 is found primarily in the nervous system, CB2 is found primarily in the gastrointestinal tract and immune cells $[4,8]$. Out of approximately 113 cannabinoids that have been identified, delta-9-tetrahydrocannabinol (THC), and cannabidiol (CBD) are best understood [10]. THC is a partial CB1 and CB2 agonist; it has a higher affinity for the CB1 receptors in the brain, which is responsible for the euphoric feeling expressed by users and may also cause anxiety and psychotic disorders $[8,11]$. On the other hand, CBD, which has antipsychotic, antianxiety, anti-nausea, antibacterial, antifungal, and anti-inflammatory effects can mitigate the effects of THC [8]. In the pancreas, both CB1 and CB2 receptors are weakly expressed in the islets of Langerhans $[2-3,8]$. When the pancreas is inflamed, the expression and activity of both $\mathrm{CB} 1$ and $\mathrm{CB} 2$ receptors are increased [8]. Wargo et al. proposed that agonism of the CB1 may play a role in the mechanism of acute pancreatitis, but to the best of our knowledge, this has not been confirmed in human studies [2]. Beyond this, little is known about how cannabinoids interact with receptors in the pancreas to cause acute pancreatitis [1,3-6]. It is possible that cannabis-induced pancreatitis may be dose and time-dependent, as seen in chronic and heavy smokers where symptoms of acute pancreatitis resolve with cessation of cannabis [1-3].

Cannabis-induced pancreatitis is considered a diagnosis of exclusion [4]. Beyond the information the patient provides, it is difficult to confirm a diagnosis of cannabis-induced pancreatitis because there are no strict guidelines to confirm the diagnosis [12]. Several case series and case reports have utilized the Naranjo Nomogram for Adverse Drug Reaction Assessment to support the diagnosis of cannabis-induced pancreatitis in their patients [2,8-13]. It may be useful for future guidelines on managing and treating cannabis-induced pancreatitis to incorporate the Naranjo score in the diagnostic criteria. With the exception of a case report where a patient presented with cannabis-induced pancreatitis at 48 years of age, other cited reports featured a pattern of symptom presentation in patients in their 20s, similar to our patient [1-2,8,12]. The 48-year-old patient presented with multiple comorbidities, including diabetes, hyperlipidemia, hypertension, and gastroesophageal reflux, which was in contrast to most of the younger patients, including our patient, who had unremarkable medical histories [12]. The most common finding in the featured cases was a dosedependent correlation between cannabis use and symptom presentation $[1,8]$. One of the limitations of this case report is that we did not obtain information about our patient's frequency amount of cannabis use. In one report, a CT finding in a 25-year-old patient showed an edematous pancreas with peripancreatic fat stranding and fluid collection, which was in contrast to our patient's unremarkable CT finding [8].

\section{Conclusions}

Cannabis is an unusual cause of acute pancreatitis. There is a paucity of information about how cannabinoids interact with receptors in the pancreas to cause acute pancreatitis. It is our hope that further research will be undertaken in this area, to facilitate an in-depth understanding of this condition.

\section{Additional Information}

\section{Disclosures}

Human subjects: Consent was obtained or waived by all participants in this study. Conflicts of interest: In compliance with the ICMJE uniform disclosure form, all authors declare the following: Payment/services info: All authors have declared that no financial support was received from any organization for the submitted work. Financial relationships: All authors have declared that they have no financial relationships at present or within the previous three years with any organizations that might have an interest in the submitted work. Other relationships: All authors have declared that there are no other relationships or activities that could appear to have influenced the submitted work.

\section{References}


1. Grant P, Gandhi P: A case of cannabis-induced pancreatitis. J Pancreas. 2004, 5:41-43.

2. Wargo KA, Geveden BN, McConnell VJ: Cannabinoid-induced pancreatitis: a case series. J Pancreas. 2007, 8:579-583.

3. Goyal H, Guerreso K, Smith B, Harper K, Patel S, Patel A, Parikh P: Severity and outcomes of acute alcoholic pancreatitis in cannabis users. Transl Gastroenterol Hepatol. 2017, 2:60. 10.21037/tgh.2017.06.03

4. Simons-Linares CR, Barkin JA, Jang S, et al.: The impact of cannabis consumption on mortality, morbidity, and cost in acute pancreatitis patients in the United States: a 10-year analysis of the national inpatient sample. Pancreas. 2019, 48:850-855. 10.1097/MPA.0000000000001343

5. Jones MR, Hall OM, Kaye AM, Kaye AD: Drug-induced acute pancreatitis: a review . Ochsner J. 2015, 15:4551.

6. Wang GJ, Gao CF, Wei D, Wang C, Ding SQ: Acute pancreatitis: etiology and common pathogenesis . World J Gastroenterol. 2009, 15:1427-1430. 10.3748/wjg.15.1427

7. Banks PA, Bollen TL, Dervenis C, et al.: Classification of acute pancreatitis-2012: revision of the Atlanta classification and definitions by international consensus. Gut. 2013, 62:102-111. 10.1136/gutjnl-2012302779

8. Pagliari D, Saviano A, Brizi MG, et al.: Cannabis-induced acute pancreatitis: a case report with comprehensive literature. Eur Rev Med Pharmacol Sci. 2019, 23:8625-8629.

9. Zhang MW, Ho RC: The cannabis dilemma: a review of its associated risks and clinical efficacy . J Addict. 2015, 707596:10.1155/2015/707596

10. Tirozzi B, Londei F, Gianani S: Depolarization block in the endocannabinoid system of the hippocampus . NeuroSci. 2020, 1:85-97. 10.3390/neurosci1020008

11. Elsohly MA, Slade D: Chemical constituents of marijuana: the complex mixture of natural cannabinoids . Life Sci. 2005, 78:539-48. 10.1016/j.lfs.2005.09.011

12. Ghazaleh S, Alqahtani A, Nehme C, Abugharbyeh A, Said Ahmed TS: A rare case of cannabis-induced acute pancreatitis. Cureus. 2019, 11:e4878. 10.7759/cureus.4878

13. Barkin JA, Nemeth Z, Saluja AK, Barkin JS: Cannabis-induced acute pancreatitis: a systematic review . Pancreas. 2017, 46:1035-1038. 10.1097/MPA.0000000000000873 